\title{
Primary and secondary dental care: how ideal is the interface?
}

\author{
A. J. Morris' ' and F. J. T. Burke ${ }^{2}$
}

\begin{abstract}
In our previous paper in this journal, we described an ideal interface between primary and secondary dental care in terms of equity, seamless care and efficiency and effectiveness. This paper examines the published evidence describing considerable ongoing problems with the interface under these headings and the ways in which those problems might be better described, quantified and addressed.
\end{abstract}

$\mathrm{T}$ he organisation of healthcare in the United Kingdom with primary care services acting as gatekeepers to secondary care is recognised as a good model for the efficient use of expensive, high technology specialist medical services. ${ }^{1}$ The same almost certainly applies to dentistry since secondary dental care services are often a limited resource and many services are heavily oversubscribed. In addition, some dental specialties are often only available from dental hospitals and are mainly provided by academics holding honorary NHS contracts. The challenge is therefore to make these services as effective as possible and a key part of this is how they relate to their referral base.

In our previous paper in this journal, ${ }^{2}$ we described an ideal interface between primary $^{3}$ (PDC) and secondary (SDC) dental care in terms of equity, seamless care, and efficiency and effectiveness. An equitable interface could be defined as one where all appropriate cases in the population are referred and there are no barriers to receiving specialist care following referral. A seamless interface could be defined as one where any treatment not available in PDC is available and accessible in SDC (and vice-versa) and where transition between different providers is easily arranged. An efficient and effective inter-

\footnotetext{
${ }^{1}$ Lecturer in Dental Public Health ${ }^{2}$ Professor of Primary Dental Care

${ }^{*}$ Correspondence to: John Morris, School of Dentistry, The University of Birmingham, St. Chad's Queensway, Birmingham B4 6NN

email:a.j.morris@bham.ac.uk

REFEREED PAPER

Received 03.04.01; Accepted 20.07.01

(C) British Dental Journal 2001; 191: 666-670
}

face is one where all referrals are appropriate, are made at the right time, patients are referred back to PDC once 'specialised' treatment is completed, patients continue to see their PDC practitioner during lengthy courses of treatment within SDC and both referral and discharge to maintenance processes are infallible. Obviously, the ideal situation does not exist in health services but keeping this framework in mind can assist with describing existing problems and their relative importance.

\section{In brief \\ - This paper examines whether the interface between primary and secondary dental care meets the ideal model described in a previous paper, using published studies as evidence \\ - The apparent mismatch between the ideal interface and that described by published studies is discussed \\ - Possible solutions to the problems described are summarised}

Hospital in-patient and out-patient waiting times continue to be a key government concern. The NHS national plan ${ }^{4}$ has announced waiting time initiatives for accident and emergency, first consultation and in-patient care. In-patient oral surgery services, being closer to other medical services than other dental specialties, fit well with this approach to defining and managing quality. However, these initiatives are not necessarily appropriate to the other secondary dental care services and they have often been somewhat marginalised because treatment is usually provided on an outpatient basis.

This paper examines the published evi- dence describing the interface under the headings of equity, seamless care, and efficiency and effectiveness, and the ways in which problems might be addressed.

\section{Equity}

Probably the most important factors in the equity of uptake of SDC are the historical variations in demand for PDC between different population groups and more recent problems of availability of the latter, leading to inequalities in surveillance and referral. The former is arguably not a problem of the interface but discussion of equity and the interface needs to take place in the context of this issue. The problem of access to surveillance and referral services is a particular and unique issue for dentistry since in medicine $98 \%$ of the population are registered with a GMP. ${ }^{1}$

Another potential problem is the scarcity of NHS SDC within a reasonable travelling distance of the population. Whilst basic oral surgery services are available at most district general hospitals, as are to a slightly lesser extent orthodontic services, this is not true of the other dental specialties which are mostly only available from the NHS through dental hospitals. Most health authorities will have a service agreement for the treatment of their residents, thus avoiding the so-called 'postcode lottery' but this can still require patients to travel considerable distances.

The situation has in the main improved little in the decade since it was described by Basker, Harrison and Ralph in $1988^{5}$ and is particularly undesirable for protracted courses of treatment involving multiple visits. The reality of this may well be to restrict the provision of care to those within a reasonable travelling distance of a dental hospital or with free time and means of transport. A study of referrals to periodontology services in Northern Ireland and North West England $^{6}$ underlined the association between the likelihood of referral and distance from the specialist.

The effect of long waiting times is also inequitable, particularly in an environment where private alternatives are available. Most 


\section{PRACTICE health policy}

consultant-led dental services appear to report increasing numbers of referrals and increasing case complexity. A recent analysis of referrals to a consultant-led periodontal service at Birmingham Dental Hospital $^{7}$ showed a 1.6-fold increase in referrals over five years from an average of 64 a month in 1992/93 to 104 a month in 1997/98. Affluent and articulate patients may be able to negotiate the potentially complex process of working their way up a waiting list, have access to personal transport when keeping appointments and if all else fails may be able to afford private treatment, essentially buying themselves off NHS waiting lists, but others may not.

An arguably less important factor may be the 'office hours only' availability of most services, creating problems for those with daytime domestic responsibilities or employers who discourage health-related absence.

The ground is laid therefore for users of secondary dental care services, with the possible exception of emergency and cancer-related oral surgery services, to be an unrepresentative distillate of the population which is not explained by variations in clinical need. A study of the use of oral surgical services in the West Midlands ${ }^{8,9}$ revealed far less use of elective oral surgery services by deprived communities, though factors such as differing experience of extractions may partly explain differing experience of third molar removal. A study of patients referred to an implant clinic in Birmingham ${ }^{10}$ also showed a highly skewed distribution. Many authors report that women form a far greater proportion of referrals for restorative care, ${ }^{5,11,12}$ presumably reflecting differing levels of demand. This apparent pattern of use is reversed for services providing extractions for children $^{13}$ and maxillofacial trauma. ${ }^{14}$

\section{Seamless care}

It is perhaps inevitable that primary and secondary dental care are not coordinated to provide a seamless service, since they are funded in different ways, operate to different rules, and care is usually provided at different locations. This failure manifests itself in two ways: the first is practitioners facing difficulty in identifying somewhere to refer patients for certain forms of care, particularly anxiety management and the restorative disciplines; the second is the problems faced by specialists in referring patients back to primary dental care at the end of lengthy specialist treatment. It is apparently an increasing problem that in the intervening time between referral and discharge NHS patients have been actively or passively deregistered because they have failed to maintain contact with their GDP, often through a misunderstanding that this was necessary. Consultant services particularly now find that they have to remind patients to maintain regular contact with their own dentist during treatment and hold information on obtaining NHS dental care for those who have fallen through the net. The extent and magnitude of these problems is unknown.

\section{Efficiency and effectiveness}

The poor quality of information sent by some PDC providers when making referrals is a long-standing problem which continues to affect the efficiency of SDC. A recent review of 161 dental consultants ${ }^{15}$ found that nearly a quarter of referral letters were inadequate and that this was a common finding across dental specialties, though consultant orthodontists tended to report an even greater proportion of inadequate referral letters.

The published research into efficiency and effectiveness tends to concentrate mainly on consultant-led studies of referral quality, there being little PDC-led research and a number of studies highlight major problems with the adequacy of the referral process. ${ }^{16,17}$ An additional issue is the lack of trained manpower to take on intermediate cases. ${ }^{18}$

Whilst in medicine there may be a tendency for hospital services to 'capture and retain' patients where there is no overt incentive to refer them back to primary care $^{19}$ the issue has not been looked at in dentistry, but is a potential problem for restorative disciplines where there may not be an obvious end point in treatment.

Lastly, the patient failing to attend for consultation or treatment apparently continues to be a major problem; it was recently announced that around one in ten patients across medical and dental specialties in England fail to keep their first out-patient appointment, ${ }^{20}$ this being a significant problem for high-cost procedures such as in-patient oral surgery. ${ }^{21}$

In summary, there are a number of barriers to the achievement of an ideal interface (Table 1). What therefore are the catalysts and what might be done to promote them?

\section{Possible solutions}

Some of the problems described are likely only to apply to certain specialties in certain parts of the UK but many, we suspect, are widespread. Before considering solutions the major requirement is for the profession to achieve a better understanding of the factors that shape the interface and whether these substantially vary by location and specialty. Undoubtedly the problems with the interface between primary and secondary dental care are multiple and complex. Individual solutions are therefore likely to be partial and may themselves be complex in operation. In some ways, the solutions can be described in terms of the reverse of the problems (Table 1). Many of these suggested initiatives have been tried in the past; some are established and operate in many areas, others are still undergoing development. The majority fall into the categories of widening access, decision support and improving efficiency though these are not neat categories and many solutions fall across categories.

\section{Widening access}

Anything which improves access to PDC and SDC is to be recommended. The development of outreach by consultant-led services is one possible solution, as is the development of NHS specialist care outside of consultant-led units, such as in some of the PDS pilots. ${ }^{22}$

Where consultant-led services are undergoing reconfiguration, the maintenance of 'spoke' services may do much to reduce the impact on access. ${ }^{23}$ In a study of GDPs in North East England, ${ }^{24}$ the majority of respondents reported a preference for consultant-led services when making referrals for restorative dentistry. The authors con- 


\section{Table I \\ Barriers and catalysts to the achievement of an ideal interface between primary and secondary dental care.}

\section{Ideal Quality Barriers \\ Equity

Scarcity of NHS SDC
providers, with increased
indirect costs for patients \\ Access/availability problems with surveillance and referral from PDC providers \\ Direct costs associated with both NHS (other than in hospitals) and private SDC \\ Indirect costs (eg travel, lost work time, child care) \\ Lack of appropriate patient demand \\ Long NHS waiting times coupled with availability of private specialist care}

\section{Seamless care Lack of co-ordination}

Efficiency \& Effectiveness between PDC and SDC

\section{Access/availability problems with SDC}

\section{Access/availability problems with PDC when referral back to PDC needed}

\section{Catalysts}

Increased coverage of PDC

Community-based NHS
SDC providers

Minimising direct and indirect costs

\section{Possible screening of} groups with poor access or inappropriately low demand

\section{Coordination to make services complementary}

Increased capacity within PDC and SDC

\section{Direct booking schemes}

Problems with availability of PDC leading to rise in acute cases, late presentation, and difficulty in referring back to primary care.

\section{Skills issues causing} inappropriate referral and non-referral

\section{Referral protocols} covering movement in both directions between PDC and SDC

Improved training for both PDC and SDC practitioners

Improvements in screening cluded that this was possibly explained by non-consultant specialist practitioners tending to work in the private sector, hence consultant services were a far cheaper option for patients. In addition they concluded that business competition with nonconsultants might be a factor.

There are however obvious barriers to promoting specialist care within a primary care setting, including skills shortage and the NHS GDS fees structure which is perceived not to reward complex care ${ }^{25}$ and in an environment of problems of availability of primary dental care there are concerns that such developments would take more dentists away from their 'core' task. For maximum benefit, any such developments need to be coordinated with consultant services $^{26}$ so that patients are referred to the most appropriate provider.

The use of selective screening as a way of stimulating demand amongst people potentially in need of specialised care but with low demand has been suggested for oral cancer but remains a complex issue with uncertain outcomes.

Lastly, the development of specialist care within the Bodies Corporate ${ }^{27}$ may act to increase capacity, though this is likely to be in the private sector.

\section{Decision support}

A commonly tried solution to the joint problems of variation in referral patterns between different primary dental care practitioners and inappropriate referrals has been the introduction of referral guidelines with or without associated training. Whilst these are undoubtedly widespread, there are few studies examining their effect. In a review of research into clinical guidelines ${ }^{28}$ only one dental research project was found, evaluating guidelines for referral to oral surgery and orthodontic services in the North West. More recently, O'Brien et. al. ${ }^{29}$ published a randomised controlled trial of orthodontic referral guidelines in the North West. Disappointingly this showed no evidence that guidelines reduced inappropriate referrals, though for methodological purposes the guidelines used were not matched with a referral proforma, which might have assisted compliance. Another 
recent study of the impact of third molar guidelines in one locality ${ }^{30}$ has suggested that they were instrumental in improving referring behaviour.

Improving decision-making may well increase referral from some PDC practitioners who were previously failing to refer appropriate cases but there is no published evidence to support this.

Developments in the use of information technology ${ }^{31}$ and the role of telemedicine $e^{32}$ have potentially much to offer here by providing eassy access to updated referral guidance and allowing an interactive discussion of cases prior to a referral decision. Notwithstanding these developments, paper-based systems may well continue to have a role. ${ }^{31,33}$

\section{Improving efficiency}

Initiatives to improve the quality of referrals as above are likely to do much to improve the efficiency of the interface. As part of this many units, particularly in orthodontics, have explicit patient acceptance criteria and may not accept referrals without evidence that the patient's needs are in line with them. The development of the index of orthodontic treatment need (IOTN) as a way of classifying treatment need has proved a useful and valid tool for such orthodontics, the development of similar tools for other specialties, whilst arguably more difficult, might prove similarly beneficial, particularly if they are backed by outcome measures.

Certainly guidelines now exist for single items of care such as implants ${ }^{34}$ and an index of restorative treatment need has recently been developed by the Royal College of Surgeons of England. The development of an 'advice only' policy in certain situations, whilst offering improved efficiency has its own pitfalls. ${ }^{24}$ The use of direct bookings or booked admissions is now being piloted in dentistry and telemedicine may also act to improve efficiency.

\section{Conclusions}

In these two papers we have set out a framework for describing the interface between primary and secondary dental care, the forces driving referral of patients to special- ists and some of the current problems. In describing apparent problems we do not mean to criticise either side of the interface and we acknowledge that the issues raised will not necessarily apply to all dental specialties in all parts of the UK. The reader must remember that we have described problems with the interface only, not every aspect of the services themselves. Many of the problems identified are backed by limited research, as are suggested solutions and our understanding of the interface is hampered by the absence of high quality activity data. ${ }^{35}$ Most research has centred around inappropriate referrals and whilst this research is important, Primary Dental Care and patientbased research potentially has much to offer, allowing insight into such issues as inappropriate non-referral. Such research will not be easy because of the relatively low incidence of cases needing referral in Primary Dental Care, though periodontology and orthodontics might be exceptions. The overriding concern should be to improve our understanding of how the interface operates, through which the profession will be in a better position to control the forces of change rather than be driven by them.

The authors are grateful to Dr I. L. Chapple for access to his referral data.

1 Bowling A, Redfern J. The process of outpatient referral and care: the experiences and views of patients, their general practitioners, and specialists. Br J Gen Prac 2000; 50: 116-120.

2 Morris A J, Burke F J T. Primary and secondary dental care; the nature of the interface. Br Dent J 2001; 191: 660-664.

3 Morris A J, White D A, Bradnock G. Primary dental care: time to revise the definition? Prim Dent Care 2000; 7: 93-96.

4 Department of Health. The NHS Plan: A plan for investment, a plan for reform. Cm 4818-I. London: The Stationary Office, 2000.

5 Basker R M, Harrison A, Ralph J P. A survey of patients referred to restorative dentistry clinics. Br Dent J 1988; 164: 105-108.

6 Linden G J, Stevenson M, Burke F J T. Variation in periodontal referral in two regions in the UK. J Clin Periodontol 1999; 26: 590-595.

7 Hamburger R. Oral health strategy. Birmingham Health Authority, January 1999.

8 Gilthorpe M S, Bedi R. An exploratory study combining hospital episode statistics with socio-demographic variables, to examine the access and utilisation of hospital oral surgery services. Community Dent Health 1997; 14: 209-213.
9 Gilthorpe M S, Wilson R C, Bedi R A Sociodemographic analysis of inpatient oral surgery: 1989-1994. Br J Oral Maxillofac Surg 1997; 35: 323-327.

10 White D A, Laird W R L, Barclay C W. Patterns of implant referral using a Geographical Information System (GIS). Proceedings of the British Society for the Study of Prosthetic Dentistry, April 2000.

11 Yemm R. Analysis of patients referred over a period of five years to a teaching hospital consultant service in dental prosthetics. $\mathrm{Br}$ Dent J 1985; 159: 304-306.

12 Callis P D, Charlton G, Clyde J S. A survey of patients seen in consultant clinics in conservative dentistry at Edinburgh Dental Hospital in 1990. Br Dent J 1993; 174: 106-110.

13 Landes D P, Bradnock G. Demand for dental extractions performed under general anaesthesia for children by Leicestershire Community Dental Service. Community Dent Health 1996; 13: 105-110.

14 Moles D R, Gilthorpe M S, Wilson R C, Bedi R. Variations in admission to hospital for head injury and assault to the head. Part 2: Ethnic group. Br J Oral Maxillofac Surg 1999; 37: 301308.

15 McAndrew R, Potts A J C, McAndrew M, Adam S. Opinions of dental consultants on the standard of referral letters in dentistry. Br Dent J 1997; 182: 22-25.

16 Burke F J T, Goodall C, Hayes F. Appropriate and inappropriate referrals to a unit of conservative dentistry. Prim Dent Care 1999; 6: 141-144.

17 O’Brien K, McComb J L, Fox N, Bearn D, Wright J. Do dentists refer orthodontic patients inappropriately? Br Dent J 1996; 181: 132-136.

18 Russell J I, Pearson A I, Bowden D E J, Wright J, O'Brien K D O. The consultant orthodontic service - 1996 survey. Br Dent J 1999; 187: 149-153.

19 Williams R. Breaking the barriers for improved glycaemic control: primary care and secondary care interface. Diabetic Med 1998; 15(Suppl. 4): S37-40.

20 Department of Health Statistical.Press notice NHS waiting list activity figures. Press release 2000/0091, 15th February 2000.

21 Thompson P J. Cancelled operations. A current problem in oral and maxillofacial surgery. $\mathrm{Br}$ Dent J 1991; 171: 244-245.

22 Kettler C J. The Bedfordshire PDS orthodontic pilot. Br J Orthod 1999; 26: 342-345.

23 BDA Central Committee for Hospital Dental Services. Reconfiguration of acute general hospital services - implications for the dentally based specialties. British Dental Association July 2000.

24 Fairbrother K J, Nohl F S A. Perceptions of general dental practitioners of a local secondary care service in restorative dentistry. Br Dent J 2000; 188: 99-102.

25 Lynn T. From a dental advisor. Dent Pract 1999; 37: 2 .

26 Langford J W, Ferguson J W. A comparison of consecutive orthodontic referrals seen by a 
consultant unit and specialist orthodontic practitioners. Br J Orthod 1995; 22: 347-353.

27 'Boots announces UK referral network'. Dent $15 / 2 / 2001$.

28 Dowie R. A review of research in the United Kingdom to evaluate the implementation of clinical guidelines in general practice. Fam Pract 1998; 15: 462-470.

29 O'Brien K, Wright J, Conboy F, et al. The effect of orthodontic referral guidelines: a randomised controlled trial. Br Dent J 2000; 188: 392-397.
30 Heath S, Worral S F, Rhead S. Audit of general dental practitioners' referral practice following the distribution of third molar guidelines. $J$ Integrated Care 2000; 4: 9.

31 Goodey R D, Brickley M R, Hill C M, Shepherd J P. A controlled trial of three referral methods for patients with third molars. Br Dent J 2000; 189: 556-560.

32 'Government funding for dentists in new referral scheme.' BDA News 2000; 13: 8 .
33 Srinivasan V, Roebuck E, Wilson E. The compliance of referral letters for GA with GDC guidelines. J Dent Res 2001; 80: 1149.

34 Faculty of Dental Surgery Guidelines for selecting appropriate patients to receive treatment with dental implants: priorities for the NHS. National Clinical Guidelines 1997 Royal College of Surgeons of England 1997.

35 Jessop N M, Kay E J, Mellor A C, Whittle J G, Jenner A. Management information failings and future requirements for dental commissioning groups. Br Dent J 2001; 190: 36-38. 